\title{
ÜBER EINEN SATZ DES HERRN SERGE BERNSTEIN.
}

\author{
VON
}

\author{
MARCEL RIESZ
}

in Sтоскноц.

(Aus zwei Briefen an Herrn G. Mittag-Leffler.)

I.

... Neulich haben wir uns über einen Satz von Herrn S. Bernstein unterhalten. Ich kann Ihnen jetzt für denselben einen ganz elementaren Beweis mitteilen, der auf der LaGRangmschen Interpolationsformel beruht.

Ich werde zunächst den zu beweisenden Satz folgendermassen aussprechen.

Es sei $f(x)$ ein Polynom von höchstens $n$-tem Grade mit beliebigen komplexen Koeffizienten

$$
f(x)=a_{0}+a_{1} x+\cdots+a_{n} x^{n} .
$$

Es bedeute $L$ das Maximum des absoluten Betrages von $f(x)$ auf der Strecke (- I, $+\mathrm{I})$. Bezeichnen wir dann mit $\xi$ einen beliebigen Punkt, der ausserhalb ${ }^{1}$ der Strecke $(-\mathrm{I},+\mathrm{x})$ liegt, so ist

$$
|f(\xi)| \leq L(A+B)^{n}
$$

wo $A$ und $B$ die Halbachsen der Ellipse bedeuten, die durch den Punkt $\xi$ geht und die Punkte - I und + I zu Brennpunkten hat.

Herr Bernstern² beweist für Polynome mit reellen Koeffizienten

$$
|f(\xi)|<L(A+B)^{n}
$$

$(n>0)$

${ }^{1}$ Der Satz bleibt richtig, wenn $\xi$ auf der Strecke $(-1,+1)$ gelegen ist, wird aber in diesem Falle trivial, da jetzt die Ellipse in die (doppelte) Strecke $(-r,+1)$ ib bergeht und $A+B=I$ wird.

S. Berssteis: (I), Sur l'ordre de la meilleure approximation des fonctions continues par des polynomes de degré donné, Mémoires publiés par la Classe des Sciences de l'Académie de Belgique, Bd. 4, 1912, S. 13-15. In dieser Arbeit wird der Satz nur für reelle $\xi$ bewiesen. Der allgemeine Beweis befindet sich in der Arbeit (II): Sur une propriété des polynomes, Bulletin de la Soc. Math. de Kharkof, Bd. 14.

Acta mathematica. 40. Imprimé le 12 juillet 1916. 
und für Polynome mit beliebigen komplexen Koeffizienten

$$
|f(\xi)|<2 L(A+B)^{n} .
$$

Ich werde Ihnen demnächst einen auf der Theorie der analytischen Funktionen fussenden Beweis mitteilen, durch welchen ich zeigen kann, dass (2) auch für Polynome mit beliebigen komplexen Koeffizienten richtig ist. Mein elementares Verfahren ergibt aber für beliebige $\xi$ nur die weniger besagende Ungleichung (I). (Wie ich am Ende dieses Briefes zeigen werde, ergibt z. B. für reelle $\xi$ die $\mathbf{L A}_{\mathbf{A}}-$ GRANGEsche Interpolationsformel sogar mehr als (2), aber nicht für ein beliebiges $\xi$ ).

Um die Interpolationsformel anwenden zu können, bestimmen wir zunächst ein Polynom $(n+I)$-ten Grades $g(x)$, das in den Punkten

$$
x_{0}=\mathrm{I}, x_{1}=\cos \frac{\pi}{n}, \ldots x_{r}=\cos \frac{r \pi}{n}, \ldots x_{n}=-\mathrm{I}
$$

verschwindet. $\mathrm{Zu}$ diesem Ende setzen wir für $-\mathrm{I} \leqq x \leqq \mathrm{I}, x=\cos \varphi$. Verändert sich nun $\varphi$ von o bis $\pi$, so beschreibt $x$ die Strecke $(+\mathrm{I},-\mathrm{I})$. Hieraus erhellt, dass das fragliche Polynom

$$
g(x)=\sin (\arccos x) \sin (n \arccos x)=G(\varphi)=\sin \varphi \sin n \varphi
$$

gesetzt werden kann. ${ }^{1}$

Nach der LAGRANGmschen Interpolationsformel ist nun

$$
f(x)=g(x) \sum_{r=0}^{n} \frac{f\left(x_{r}\right)}{\left(x-x_{r}\right) g^{\prime}\left(x_{r}\right)}
$$

Um diesen Ausdruck explizit zu berechnen, schreiben wir

$$
g^{\prime}\left(x_{r}\right)=\left[\frac{d}{d \varphi}(\sin n \varphi \sin \varphi) \frac{d \varphi}{d x}\right]_{\varphi=\varphi_{r}}=-\left[\frac{n \cos n \varphi \sin \varphi+\cos \varphi \sin n \varphi}{\sin \varphi}\right]_{\varphi=\varphi_{r}}
$$

Hieraus erhalten wir

und

$$
g^{\prime}\left(x_{r}\right)=(-\mathrm{I})^{r+1} n \quad(0<r<n)
$$

1 Dass $g(x)$ wirklich ein Folynom $(n+1)$-ten Grades in $x=\cos \varphi$ ist, folgt $z$. B. aus der Formel

$$
g(x)=\frac{\sqrt{\mathrm{I}-x^{2}}}{2 i}\left[\left(x+i \sqrt{\mathrm{I}-x^{2}}\right)^{n}-\left(x-i \sqrt{\mathrm{I}-x^{2}}\right)^{n}\right] .
$$

Hier kann der Quadratwurzel irgend einer der beiden möglichen Werte beigelegt werden, der aber dann an allen drei Stellen derselbe sein muss. 
Mithin

$$
\begin{gathered}
g^{\prime}(\mathrm{I})=-2 n, \\
g^{\prime}(-\mathrm{I})=(-\mathrm{I})^{n+1} 2 n .
\end{gathered}
$$

(3) $f(x)=\frac{g(x)}{n}\left(-\frac{f(\mathrm{I})}{2(x-\mathrm{I})}+(-\mathrm{I})^{n+1} \frac{f(-\mathrm{I})}{2(x+\mathrm{I})}+\sum_{r=1}^{n-1}(-\mathrm{I})^{r+1} \frac{f\left(x_{r}\right)}{x-x_{r}}\right)$.

Es sei nun $\xi$ ein beliebiger spezieller Wert von $x$, der ausserhalb der Strecke $(-I,+I)$ liegt. Wir können dann nach dem Vorgehen von Herrn Bernstein

$$
\xi=\cos (a+b i)=\frac{e^{b}+e^{-b}}{2} \cos a-i \frac{e^{b}-e^{-b}}{2} \sin a
$$

setzen. Da Kosinus eine gerade Funktion ist, können wir hierbei $b>0$ wählen. Dann zeigt aber die Formel (4) folgendes. Ist $a+b i$ einer der Werte von are $\cos \xi$ und ist $b>0$, so ist $\xi$ auf einer Ellipse belegen, die die Punkte $-\mathrm{I}$ und + I zu Brennpunkten hat und deren Halbachsen $\frac{e^{b}+e^{-b}}{2}$ und $\frac{e^{b}-e^{-b}}{2}$ sind. Die Summe dieser Halbachsen ist natürlich $e^{b}$.

Wir schätzen nun $|\sin (a+b i)| a b$.

$$
\begin{aligned}
|\sin (a+b i)|=\mid \frac{e^{b}+e^{-b}}{2} \sin a+i \frac{e^{b}-e^{-b}}{2} & \cos a \mid= \\
& =\frac{I}{2} \sqrt{e^{2 b}+e^{-2 b}-2 \cos 2 a} \leqq \frac{e^{b}+e^{-b}}{2}-<e^{b} ;
\end{aligned}
$$

ebenso

$$
|\sin n(a+b i)| \leq \frac{e^{n b}+e^{-n b}}{2}<e^{n b}
$$

Mithin

(5) $|g(\xi)|=|\sin (n \arccos \xi) \sin (\arccos \xi)|=|\sin n(a+b i) \sin (a+b i)|<e^{(n+1) b}$.

Wir bezeichnen nun mit $\delta$ das Minimum des Abstandes von $\xi$ zu den Punkten der Strecke $(-I,+I)$. Dann erhalten wir aus (3) und aus (5) zunächst folgende sehr grobe Abschätzung

$$
|f(\xi)| \leqq \frac{e^{(n+1) b}}{n} \frac{L n}{\delta}=L \frac{e^{(n+1) b}}{\delta} .
$$

Dieses Resultat wenden wir jetzt auf das Polynom $(f(x))^{m}$ an, wo $m$ eine beliebige positive ganze Zahl bedeutet. Wir erhalten 


$$
|f(\xi)|^{m} \leqq L^{m} \frac{e^{(m n+1) b}}{\delta}
$$

also

$$
|f(\xi)| \leqq L e^{n b} \sqrt[m]{\frac{e^{b}}{\delta}}
$$

Lassen wir nun $m$ ins Unendliche wachsen, so ergibt sich

$$
|f(\xi)| \leqq L e^{n b} \cdot 1
$$

W. z. b. w.

Für reelle Werte von $\xi$ ergibt die Interpolationsformel folgende Verallgemeinerung eines Satzes von TchевуснеFF. ${ }^{2}$

Unter den am Anfange dieses Briefes gemachten Voraussetzungen ist für jeden reellen Wert $\xi$, der ausserhalb der Strecke $(-\mathrm{I},+\mathrm{I})$ liegt,

$$
|f(\xi)| \leqq L|\cos n(\arccos \xi)|,^{3}
$$

das Gleichheitszeichen ausgeschlossen, wenn das Polynom $f(x)$ nicht von der Form $L e^{i \Theta} \cos (n \operatorname{arc} \cos x)$ ist, wobei $\Theta$ eine beliebige reelle Zahl bedeutet.

In der Tat ergibt die Formel (3) für ein beliebiges $x$

$$
|f(x)| \leqq \frac{L|g(x)|}{n}\left(\frac{\mathrm{I}}{2|x-\mathrm{I}|}+\frac{\mathrm{I}}{2|x+\mathrm{I}|}+\sum_{r=1}^{n-1} \frac{\mathrm{I}}{\left|x-x_{r}\right|}\right) .
$$

${ }^{1}$ Man kann einen ähnlichen Beweis und dasselbe Resultat erhalten, wenn man in $f(x)$ $x=\cos \varphi$ setzend, $f(\cos \varphi)$ in eine endliche Fouriersche Reihe entwickelt, $\mathrm{d} . \mathrm{h}$.

$$
f(\cos \varphi)=\sum_{x=0}^{n} \alpha_{x} \cos x \varphi
$$

schreibt und dann die Koeffizienten $a_{x}$ abschätzt; man erhält für $|f(\xi)|$ zunächst eine grobe Abschätzung, die dann, wie oben, verfeinert werden kann.

Den Kunstgriff, eine grobe Abschätzung auf eine beliebig hohe Potenz einer Funktion anzuwenden, um daraus eine genauere Abschätzung für die Funktion selbst herzuleiten, entnehme ich einem Schreiben von Herrn Landau. Herr Landa wendet daselbst den genannten Kunstgriff auf ein von dem unsrigen völlig verschiedenes Problem an.

2 'TCHEBYCHEFF: Sur les fonctions qui s'écartent peu de zéro pour certaines valeurs de la variable, Werke, Bd. 2, S. 335-356. Vergl. auch Bernstein (I), S. I3-I4. Tschebrcherf und Bernstein beweisen diesen Satz nur für Polynome mit reellen Koeffizienten und zeigen in diesem Falle, dass das Gleichheitszeichen nur für das Polynom $f(x)= \pm L \cos (n$ arc $\cos x)$ bestehen kann. In seiner späteren Arbeit (II) zeigt Herr Bersstein, dass für Polynome mit reellen Koeffizienten die Ungleichung (6) für beliebige komplexe $\xi$ gilt, die der Bedingung $|\xi| \geqq \mathrm{I}$ genügen.

Dass diese Abschätzung genauer ist als (I), folgt z. B. aus der Ungleichung $|\cos n(a+b i)|=$ $=\frac{1}{2} \sqrt{e^{2 n b}+e^{-2 n b}+2 \cos 2 n a} \leqq \frac{e^{n b}+e-n b}{2}<e^{n b}$. Vergl. BerksteIN (I), S. I4. 
Setzen wir andererseits in (3) statt $f(x)$ die Funktion $\cos (n \operatorname{arc} \cos x)$ ein und beachten, dass $\cos \left(n \operatorname{arc} \cos x_{r}\right)=(-I)^{r}$ ist, so erhalten wir

$$
\cos (n \arccos x)=-\frac{g(x)}{n}\left(\frac{I}{2(x-I)}+\frac{I}{2(x+I)}+\sum_{r=1}^{n-1} \frac{I}{x-x_{r}}\right) .
$$

Bedeutet nun $\xi$ einen beliebigen reellen Wert, der entweder $>$ I oder $<-$ I ist, und setzen wir diesen Wert in (7) und (8) ein, so haben alle Glieder der Klammer in (8) dasselbe Vorzeichen, mithin ist der absolute Betrag dieser Klammer gleich dem Werte der Klammer in (7), folglich ist die rechte Seite von ( 7$)$ gleich $L|\cos (n \operatorname{arc} \cos \xi)|$. Also endlich

$$
|f(\xi)| \leq L|\cos (n \operatorname{arc} \cos \xi)| .
$$

Man sieht auch unmittelbar (da $g(\xi)$ für die in Betracht kommenden Werte nicht verschwindet), dass das Gleichheitszeichen in (7), also auch in (6), nur dann bestehen kann, wenn die Werte $f\left(x_{r}\right)$ abwechselnd gleich $\pm e^{i \theta} L$ sind. Dann ist aber, wie leicht ersichtlich,

$$
f(x)= \pm e^{i \theta} L \cos (n \arccos x) .
$$

Es ist einleuchtend, dass es in dem zuletzt bewiesenen Satze genügt, statt $|f(x)| \leqq L$, für $-\mathrm{I} \leqq x \leqq \mathrm{I}$, nur $\left|f\left(x_{\mathrm{r}}\right)\right| \leqq L(r=0, \mathrm{I}, \ldots n)$ vorauszusetzen.

Islinge, den 23. Mai x9I5.

II.

...Wie ich schon in meinem vorigen Briefe angedeutet habe, lässt sich, für $n>0$, die Ungleichung (I) jenes Briefes durch die schärfere Ungleichung (2) ersetzen; d. h. unter den dortigen Voraussetzungen gilt für einen beliebigen Punkt $\xi$, der ausserhalb der Strecke $(-I,+I)$ liegt, die Ungleichung

$$
|f(\xi)|<L(A+B)^{n} \text {. }
$$

Der Beweis beruht auf einfachen funktionentheoretischen Betrachtungen, welche die wahre Grundlage des Satzes zu bilden scheinen.

Man denke sich die Ebene längs der Strecke $(-I,+I)$ aufgeschnitten und bilde in dieser aufgeschnittenen Ebene die Funktion

$$
\psi(z)=z+\sqrt{z^{2}-1} .
$$


Es wird derjenige Zweig der Quadratwurzel genommen, der für alle reelle $z>\mathrm{I}$ positiv ist. Dadurch ist $\psi(z)$ in der aufgeschnittenen Ebene eindeutig bestimmt.

Wir beweisen nun den folgenden (übrigens als bekannt zu betrachtenden) Hilfssatz:

Auf jeder Ellipse, mit den Brennpunkten $-\mathrm{I}$ und $+\mathrm{I}$ und den Halbachsen $A$ und $B$, ist $|\psi(z)|$ konstant und ist gleich $A+B$. Speziell ist auf der doppelten Strecke $(-\mathrm{I},+\mathrm{I})|\psi(z)|=\mathrm{I}$.

Bedeutet $z$ einen Punkt der genannten Ellipse, so kann $z=A \cos \varphi+i B \sin \varphi$ gesetzt werden. Ausserdem ist $A^{2}-B^{2}=\mathrm{I}$. Also

$$
\begin{aligned}
\psi(z) & =A \cos \varphi+i B \sin \varphi+\sqrt{A^{2} \cos ^{2} \varphi+2 i A B \cos \varphi \sin \varphi-B^{2} \sin ^{2} \varphi-I} \\
& =A \cos \varphi+i B \sin \varphi+\sqrt{A^{2} \cos ^{2} \varphi+2 i A B \cos \varphi \sin \varphi-B^{2} \sin ^{2} \varphi-A^{2}+B^{2}} \\
& =A \cos \varphi+i B \sin \varphi+\sqrt{B^{2} \cos ^{2} \varphi+2 i A B \cos \varphi \sin \varphi-A^{2} \sin ^{2} \varphi} .
\end{aligned}
$$

Der Wert der Quadratwurzel ist $\pm(B \cos \varphi+i A \sin \varphi)$. Da demjenigen Punkte der Ellipse, der auf der positiven reellen Achse liegt, der Wert $\varphi=0$ entspricht, muss das Vorzeichen + genommen werden. Mithin ist

und

$$
\psi(z)=(A+B) e^{i \varphi}
$$

$$
|\psi(z)|=A+B
$$

Die Doppelstrecke $(-I,+I)$ kann als diejenige Ellipse aufgefasst werden, für welche $A=\mathrm{I}$ und $B=\mathrm{o}$ wird, folglich übergeht hier die obige Gleichung in $|\psi(z)|=I$. Dies folgt übrigens auch daraus, dass auf dieser Strecke $z$ reell und $\sqrt{z^{2}-\mathrm{I}}= \pm i \sqrt{\mathrm{I}-z^{2}}$ rein imaginär ist.

Wir betrachten nun in der aufgeschnittenen Ebene die Funktion

$$
g(z)=\frac{f(z)}{(\psi(z))^{n}}
$$

Diese Funktion ist in jedem inneren Punkte der aufgeschnittenen Ebene (auch im Unendlichen) regulär analytisch, mithin erreicht ihr absoluter Betrag sein Maximum auf der Grenze, d. h. auf den beiden Rändern der Doppelstrecke $(-I,+I)$. Hier ist aber der absolute Betrag des Nenners gleich I, und der absolute Betrag des Zählers ist nach unserer Voraussetzung $\leqq L$. Hieraus folgt schon, dass in der ganzen Ebene $|g(z)| \leq L$ ist. Da aber der Zähler von $g(z)$ ein Polynom, und der Nenner, für $n>0$, kein Polynom ist, ist $g(z)$ keine Konstante. Mithin ist in jedem Punkte, der ausserhalb der Strecke $(-I,+I)$ liegt, 
d. h.

$$
|g(z)|=\left|\frac{f(z)}{(\psi(z))^{n}}\right|<L
$$

$$
|f(z)|<L(A+B)^{n} \text {. W. z. b. w. }{ }^{1}
$$

Unser Beweis lässt sich auch leicht auf den Fall übertragen, wo $f(z)$ ein Polynom in $z$ und $\sqrt{z^{2}-1}$ vom Gesamtgrade $\leqq n$ ist. Hierdurch erhält man, wenn man noch $z=\cos x$ setzt, einen Satz über trigonometrische Polynome, auf welchen ich aber hier nicht näher eingehen will, da er auch aus unseren späteren Betrachtungen folgt.

Ich sagte oben, dass der angewandte Hilfssatz als bekannt zu betrachten ist. In der Tat bildet die Funktion $x=\psi(z)$ einen Zweig der Umkehrung der Funktion

$$
z=\frac{\mathrm{I}}{2}\left(x+\frac{\mathrm{I}}{x}\right)
$$

Die Funktion (9) und ihre Umkehrung werden z. B. im zweiten Bande des Traité d'Analyse von Herrn Picard eingehend behandelt. (Vergl. 2. Aufl. S. 3I4-3I7.) Die Funktion (9) bildet jeden um den Anfangspunkt beschriebenen Kreis, dessen Halbmesser $r>_{I}$ ist, auf eine Ellipse $a b$, deren Halbachsen $A$ und $B$ der Gleichung $A+B=r$ genügen, und deren Brennpunkte $-\mathrm{I}$ und $+\mathrm{I}$ sind. Der Kreis mit dem Halbmesser $I$ wird auf die doppelte Strecke $(-I,+I)$ abgebildet. In diesen Tatsachen ist der obige Hilfssatz enthalten.

Jeder Kreis, dessen Halbmesser $r<I$ ist, wird durch die Funktion (9) wieder auf eine Ellipse abgebildet, deren Halbachsen die Gleichung $A-B=r$ erfüllen und deren Brennpunkte ebenfalls $-I$ und $+I$ sind. Die $x$-Ebene wird also durch die Funktion (9) auf die doppelte $z$-Ebene abgebildet. Die Umkehrfunktion dieser Abbildung hat folglich zwei Werte, die durch die Formeln

und

$$
x_{1}=z+\sqrt{z^{2}-1}
$$

$$
x_{2}=z-\sqrt{z^{2}-I}
$$

dargestellt werden. Natürlich ist $x_{1} x_{2}=\mathrm{I}$.

1 Dieser Beweis, den mir Herr Marcel Riesz seiner Zeit mitteilte, wurde von mir in zwei kleineren Aufsătzen Über einen Satz des Herrn Serge Berssters, Sitzungsberichte der Kgl. Bayer. Akad. d. Wissensch., Tahrg. 1915, S. 419-424" und s Sur un théorème de M. Serge Bern steın, The Tohôku Math. Journal, Vol. 9, Nos. I, 2, I916, S. I-6, obne andere Bemerkung wiedergegeben, als mit einem Hinweis auf den obigen Brief. Durch unvorhergesehene Umstande wurde jedoch das Drucken desselben derart verzögert, dass meine Aufsätze früher als der Brief erschienen. Hierdurch könnte das Missverständnis aufkommen, der Beweis stamme von mir. Dies ist aber, wie ich hier ausdrücklich betonen will, durchaus nicht der Fall.

G. MittaG-LeffleR. 
Um das grosse Interesse der betrachteten Abbildung zu zeigen, will ich hier einen Satz aussprechen, der aus den angeführten Betrachtungen bei Picard unmittelbar folgt.

Jede Funktion, die ausserhalb der Strecke $(-I,+I)$ regulär analytisch ist, kann in eine Reihe der Form

$$
\sum_{n=0}^{\infty} a_{n}\left(z-\sqrt{z^{2}-\mathrm{I}}\right)^{n}=\sum_{n=0}^{\infty} a_{n}\left(z+\sqrt{z^{2}-\mathrm{I}}\right)^{-n}
$$

entwickelt werden.

Dieser Satz beruht eben auf der Tatsache, dass die Funktion $z-\sqrt{z^{2}-1}=$ $=\frac{I}{z+\sqrt{z^{2}-I}}$ in der aufgeschnittenen Ebene regulär analytisch ist, und diese Ebene auf das Innere des Einheitskreises abbildet.

Der zuletzt ausgesprochene Satz gestattet eine recht interessante Anwendung. Herr HAAR hat nämlich den folgenden merkwürdigen Entwicklungssatz gefunden. (Über analytische Funktionen mit singulärer Linie, Göttinger Nachrichten, I9r4, Heft I, S. $\left.x_{5}-\mathrm{r} 23.\right)$

Sei $F(z)$ eine Funktion der komplexen Veränderlichen $z$, die ausserhalb des doppelpunktlosen analytischen Kurvenstückes $k$ überall regulär analytisch ist. Allsdann existieren unendlich viele, längs der Kurve $k$ definierte und nur von dieser Kurve abhängige, stetige Funktionen $f_{1}(z), f_{2}(z), \cdots, f_{n}(z), \cdots$ so, dass für jede Stelle $z$, die ausserhalb der Kurve liegt, die unendliche Reihe

$$
F(\infty)+a_{1} \int_{(k)} \frac{d f_{1}(\zeta)}{\zeta-z}+a_{2} \int_{(k)} \frac{d f_{2}(\zeta)}{(\zeta-z)^{2}}+\cdots+a_{n} \int_{(k)} \frac{d f_{n}(\zeta)}{(\zeta-z)^{n}}+\cdots
$$

konvergiert und die Funktion $F(z)$ darstellt; hierbei bedeuten die $a_{n}$ Konstanten, und die Integrale sind im Stieltumsschen Sinne genommen.

Herr HaAR beweist dies durch eine konforme Abbildung auf Grund eines C. NeUmansschen Theorems über Entwicklungen nach Kugelfunktionen zweiter Art. Es ist nun leicht zu zeigen, dass, wenn man statt dieses Entwicklungssatzes den obigen anwendet, die STIELTJEsschen Integrale vermieden werden können. Es sei in der Tat $\varphi(z)$ die von Herrn HAAR angewandte Funktion, welche die durch die Kurve $k$ aufgeschnittene Ebene auf eine durch die Strecke $(-\mathrm{I},+\mathrm{I})$ aufgeschnittene Ebene konform abbildet, wobei die unendlich fernen Punkte der beiden Ebenen einander entsprechen mögen. Dann ist nach dem obigen Satz, wenn wir

setzen,

$$
\beta(z)=\varphi(z)-\sqrt{(\varphi(z))^{2}-1}
$$




$$
F(z)=F(\infty)+\sum_{n=1}^{\infty} a_{n}(\beta(z))^{n}
$$

Da nun die Funktion $\beta(z)$ in der ganzen durch die Kurve $k$ aufgeschnittenen Ebene stetig ist und im Unendlichen verschwindet, so können wir schreiben

$$
(\beta(z))^{n}=\frac{\mathrm{I}}{2 i \pi} \int \frac{(\beta(\zeta))^{n}}{\zeta-z} d \zeta
$$

wobei das Integral über die beiden Ränder der Kurve $k$ erstreckt wird und zwar so, dass die aufgeschnittene Ebene zur linken liegt. Integriert man dann nach dem Vorgehen von Herrn HAAR $(n-\mathrm{r})$-mal partiell, so müssen die total integrierten Glieder verschwinden, da die Funktion $(\beta(z))^{n}$ im Unendlichen von der $n$-ten Ordnung verschwindet. Mithin erhält man

$$
(\beta(z))^{n}=\int \frac{\gamma_{n}(\zeta)}{(\zeta-z)^{n}} d \zeta
$$

Jedem Punkte $\zeta$ der Kurve $k$ (ausser den Endpunkten) entsprechen in der aufgeschnittenen Ebene zwei Punkte $\zeta^{\prime}$ und $\zeta^{\prime \prime}$. Setzen wir $g_{n}(\zeta)=\gamma_{n}\left(\zeta^{\prime}\right)-\gamma_{n}\left(\zeta^{\prime \prime}\right)$ und in den Endpunkten $g_{n}(\zeta)=0$, so erhalten wir

$$
(\beta(z))^{n}=\int_{(k)} \frac{g_{n}(\zeta)}{(\zeta-z)^{n}} d \zeta
$$

Aus (I2) und (13) folgt nun die Darstellung

$$
F(z)=F(\infty)+\sum_{n=1}^{\infty} a_{n} \int_{(k)} \frac{g_{n}(\zeta)}{(\zeta-z)^{n}} d \zeta,
$$

wobei die Funktionen $g_{n}(\zeta)$ auf der Kurve $k$ stetig (und ausser den Endpunkten sogar regulär analytisch) sind.

Es sei noch bemerkt, dass die Funktionen $f_{n}(\zeta)$ in der Entwicklungsformel von Herrn $\mathrm{H}_{\mathbf{A A R}}$ von der zweiten angefangen eine stetige Ableitung besitzen, wie das aus dem Beweise von Herrn HaAr hervorgeht. Man könnte also auch in der Formel (II) für $n=2,3, \ldots$

$$
\int_{(k)} \frac{d f_{n}(\zeta)}{(\zeta-z)^{n}}=\int_{(k)} \frac{f_{n}^{\prime}(\zeta)}{(\zeta-z)^{n}} d \zeta=\int_{(k)} \frac{g_{n}(\zeta)}{(\zeta-z)^{n}} d \zeta
$$

Acta mathematica. 
schreiben. Die Einheitlichkeit der Darstellung würde aber dann verloren gehen, da $f_{1}(\zeta)$ keine stetige Ableitung besitzt.

Zuletzt will ich noch Ihre Aufmerksamkeit auf einige Sätze lenken, die in denselben Gedankenkreis gehören, wie der BeRnsternsche Satz.

Aus dem, was wir oben über die Abbildung $z=\frac{I}{2}\left(x+\frac{I}{x}\right)$ sagten, erhellt sofort, dass der Bernsteinsche Satz mit dem folgenden Satze gleichwertig ist:

Es sei $h(x)=\sum_{k=0}^{n} a_{k}\left(x+\frac{\mathbf{I}}{x}\right)^{k}$ auf dem Einheitskreise dem absoluten Betrage nach $\leqq L$; dann ist im Inneren des Einheitskreises $|h(x)|<L|x|^{-n}$ und im Äusseren des Einheitskreises $|h(x)|<L|x|^{n}$.

Dieser Satz bedarf ja kaum eines Beweises. Er folgt unmittelbar aus der Tatsache, dass $h(x) x^{n}$ innerhalb und $h(x) x^{-n}$ ausserhalb des Einheitskreises regulär analytisch sind.

Derselbe Gedankengang führt zu dem folgenden allgemeineren Satze:

Ist $h(x)=\sum_{k=-m}^{n} a_{l} x^{k}$ auf dem Einheitskreise dem absoluten Betrage nach $\leqq L$, so ist innerhalb des Einheitskreises $|h(x)| \leq L|x|^{-m}$ und ausserbalb des Einheitskreises $|h(x)| \leqq L|x|^{n}$. Das Gleichheitszeichen ist ausgeschlossen, wenn $h(x)$ nicht $=L e^{i \theta} x^{-m}$ bzw. $=L e^{i \theta} x^{n}$ ist, hierbei bedeutet $\Theta$ eine beliebige reelle Zahl. (Man kann selbstredend den Satz dadurch verallgemeinern, dass man die Anzahl der Glieder in irgendeiner der beiden Richtungen ins Unendliche wachsen lässt, vorausgesetzt dass die unendliche Reihe auf dem Einheitskreise z. B. gleichmässig konvergiert.)

Setzt man in diesem Satze $x=e^{s}$, so erhält man das folgende Ergebnis:

Ist $H(s)=\sum_{k=-m}^{n} a_{k} e^{k s}$ auf der imaginären Achse dem absoluten Betrage nach $\leqq L$, so ist für $R(s)>0$ (wo $R(s)$ den reellen Teil von $s$ bedeutet) $|H(s)| \leq L e^{n R(s)}$ und für $R(s)<0,|H(s)| \leq L e^{-m R(s)}$ u. s. w. ${ }^{1}$

All dies kann auch auf Ausdrücke von der Form $H(s)=\sum_{k=0}^{n} a_{k} e^{\lambda_{k} s}$ übertragen werden. Hierbei bedeuten die $\lambda_{k}$ reelle Grössen, die den Ungleichungen

1 Setzt man in diesem Satze $s=i \alpha$, so kann man $H(s)=H(i \alpha)$ in der Form $\sum_{k=0}^{N}\left(A_{k} \cos k \alpha\right.$ $+B k \sin k a)$ schreiben. Das obige kann also auch als ein Satz über trigonometrische Polynome ausgesprochen werden. 
$-\infty<\lambda_{0}<\lambda_{1}<\ldots<\lambda_{n}<+\infty$ genügen. Setzt man dann voraus, dass auf der imaginären Achse $|H(s)| \leqq L$ ist, so folgt auf Grund eines bekannten PhragménLiNDELöFschen Satzes, dass für $R(s)>0,|H(s)| \leq L e^{\lambda_{n} R(s)}$ und für $R(s)<0$, $|H(s)| \leqq L e^{\lambda_{0} R(s)}$ ist. Hierbei ist auch das Gleichheitszeichen auszuschliessen, wenn $H(s)$ nicht $=L e^{i \theta} e^{\lambda_{n} s}$ bzw. $=L e^{i \theta} e^{\lambda_{0 s}}$ ist, wobei $\Theta$ wieder eine beliebige reelle Konstante bedeutet.

Für Integrale $\int_{u}^{v} a(\tau) e^{\tau s} d \tau$, oder allgemeiner für Ausdrücke von der Form
$\int_{u}^{v} e^{\tau s} d A(\tau)$, wo $A(\tau)$ eine beliebige Funktion beschränkter Schwankung bedeuten
kann, gilt ein ähnlicher Satz. (Dieser letzte Ausdruck umfasst natürlich die
beiden vorangehenden.)
Diese Sätze enthalten ja eigentlich nichts neues; ich wollte eben nur ihren
Zusammenhang mit dem schönen Satze von Herrn Bersstein betonen. Islinge, den 25. Mai 1915.

Marcel Riesz. 